\title{
Caracterización de Planta para un Cuadricóptero
}

\section{Plant Characterization for a Quadrocopters}

Presentación:10/09/2018

Aprobación: 10/10/2018

\section{Rodrigo Cancilieri}

Facultad Regional Buenos Aires, Universidad Tecnológica Nacional -Argentina rcancilieri@est.frba.utn.edu.ar

\section{Julián A. Chiapparo}

Facultad Regional Buenos Aires, Universidad Tecnológica Nacional -Argentina jchiapparo@est.frba.utn.edu.ar

\section{Diego E. Vommaro}

Facultad Regional Buenos Aires, Universidad Tecnológica Nacional -Argentina vommaro@est.frba.utn.edu.ar

\section{Sebastián Verrastro}

Facultad Regional Buenos Aires, Universidad Tecnológica Nacional -Argentina sverrastro@frba.utn.edu.ar

\section{Resumen}

Se realizaron los ensayos pertinentes para lograr una caracterización de planta de un cuadricóptero, generando los valores de constantes necesarios para la programación de los componentes electrónicos. Se explica el motivo de cada ensayo y los resultados y conclusiones.

Palabras clave: drone; uav; ensayos.

\begin{abstract}
The pertinent tests were carried out to achieve a quadcopter plant characterization, generating the values of constants necessary for the electronic components programming. The reason for each test and the results and conclusions are explained.
\end{abstract}

Keywords: drone; uav; testing. 


\section{Introducción}

El control preciso de un drone o UAV (Unmanned Aerial Vehicule) requiere del conocimiento de las respuestas físicas de la planta. Por lo tanto, los ensayos para predecir las fuerzas que se generarán en los actuadores son de suma importancia.

Para simplificar la construcción de modelos dinámicos de sistemas inestables se trabaja con las ecuaciones físicas y los parámetros se obtienen de los modelos $\mathrm{CAD}$, salvo los parámetros de la dinámica de los actuadores que deben ser identificados mediante experimentaciones (Bouabdallah, 2007).

Este enfoque se hace presente en reconocidos trabajos pero los parámetros son presentados sin mostrar detalles acerca de su identificación (Bouabdallah, 2007) (Raffo, 2011).

Los parámetros necesarios de los actuadores son el factor de empuje y el factor de torque propio de los actuadores. Además, se necesita la matriz de momentos de inercia del cuerpo completo del cuadricóptero (Pounds, 2007).

Para la identificación del factor de empuje y el factor de torque se tienen soluciones del tipo banco de palanca (Kharsansky, 2013) (Schermuck, 2012). El factor de empuje también puede ser hallado montando al conjunto de forma que el aire impulsado sea atrapado por una caja, la cual se encuentra apoyada sobre una balanza, y entonces obtiene la medición de la fuerza del aire impulsado. El factor de torque también puede ser hallado colocando el conjunto conectado al extremo de su soporte del frame y lo utiliza como péndulo. Como al generar un torque, el péndulo varía su posición, puede obtenerse el factor buscado conociendo el peso del motor (Tavares, 2014).

La matriz de momentos de inercia se puede obtener del modelo CAD pero también es posible mediante una experiencia física del tipo Péndulo Bifilar (Kharsansky, 2013) (Schermuck, 2012).

Existe otro parámetro importante a obtener y es la constante de tiempo de los actuadores. En ocasiones no es tenida en cuenta debido a que la dinámica del cuadricóptero gobernada por su momento de inercia es dominante frente a la dinámica de los motores eléctricos que propulsan las hélices. Para llevar a cabo esta simplificación es necesario obtener su valor y compararlo con el momento de inercia del vehículo. La experiencia consiste en excitar al actuador con un escalón en el punto de trabajo y observar la dinámica con la que alcanza la velocidad final. Existen variantes en cómo medir la velocidad de los motores instante a instante. En algunos trabajos se midió con un tacómetro óptico (Kharsansky, 2011), y en otros con un micrófono cercano al aspa del actuador (Schermuk, 2012).

Teniendo los parámetros cuantificados y un sistema de control diseñado listo para correr, pude ser necesario llevar a cabo una serie de ensayos preliminares antes de un vuelo real. Estos ensayos ocurren sobre bancos de pruebas específicamente diseñados para evaluar diferentes aspectos del vehículo. Se trata de fijar algunos grados de libertad y de esta forma ejercitar al sistema de control con un 
grupo reducido de variables a estabilizar.

Este trabajo busca encontrar los métodos de caracterización de los parámetros físicos de la planta de un cuadricóptero para ser usados en el diseño del sistema de control de altitud. Además, se presentan los bancos de prueba que permiten ensayar al sistema de control en forma incremental, ideal para primeros desarrollos. Todos los bancos utilizados a lo largo del trabajo son de diseño y construcción propia.

\section{Marco teórico}

\section{Dinámica de planta}

Un cuadricóptero utiliza el empuje generado por los conjuntos motor-hélice, a los que llamaremos actuadores, para sustentarse en el aire, así como para lograr el desplazamiento.

Los actuadores se encuentran ubicados de forma simétrica respecto del centro de masa de la planta, y cada uno debe generar un empuje de igual intensidad al momento en que el drone se mantiene en un punto fijo, de modo que el empuje total compense la acción de la gravedad. Esta situación es conocida como hovering. Es importante observar que cada actuador genera, al mismo tiempo, un empuje y un torque paralelos, de modo que su sentido de giro es alternado entre actuadores (figura 1), para que los torques de igual valor y signo opuesto se cancelen, logrando que el drone se mantenga en su posición de hovering sin girar sobre su propio eje vertical (Kharsansky, 2013).

Para definir el sentido de las fuerzas y momentos aplicados sobre el cuerpo, es necesario determinar el sentido de giro de los motores y su ubicación relativa al sistema de referencia del cuerpo. En la figura 2 se puede observar la definición utilizada.

El modelo utilizado en este trabajo posee las siguientes asunciones:

1.La estructura es rígida.

2.La estructura es simétrica.

3.Las hélices son rígidas.

4.El centro de gravedad y el origen del vehículo son coincidentes.

5.El empuje generado por los actuadores es proporcional al cuadrado de la velocidad de rotación de las hélices.

Además, se considera a la influencia del efecto giroscópico causado por la rotación del cuerpo rígido y las hélices menos importantes que la acción de los motores. El resultado es un modelo simplificado del drone de segundo orden representado en las ecuaciones (1), (2) y (3) (Bouabdallah at el, 2007). 


$$
\begin{aligned}
& \ddot{\phi}=\frac{l}{I_{x x}} U_{1} \\
& \ddot{\theta}=\frac{l}{I_{y y}} U_{2} \\
& \ddot{\psi}=\frac{1}{I_{z z}} U_{3}
\end{aligned}
$$

En la tabla 1 se detallan los parámetros del modelo. Cabe aclarar que las actuaciones $\mathrm{U}_{1}$ y $\mathrm{U}_{2}$ corresponden a los empujes generados por los actuadores, y $\mathrm{U}_{3}$ representa el torque.

Finalmente, si se asume que el amortiguamiento de los actuadores es despreciable frente al momento de inercia de la planta, la relación entre las actuaciones y la velocidad de giro de los motores quedan definidas por la ecuación (4), (5) y (6).

$$
\begin{aligned}
& U_{1}=b^{\prime}\left(\Omega_{4}^{2}-\Omega_{2}^{2}\right) \\
& U_{2}=b^{\prime}\left(\Omega_{3}^{2}-\Omega_{1}^{2}\right) \\
& \left.U_{3}=d^{\prime}\left(\Omega_{2}^{2}+\Omega_{4}^{2}-\Omega_{1}^{2}-\Omega_{3}^{2}\right)\right)
\end{aligned}
$$

Generalmente, los actuadores se los suele comandar con una señal del tipo PWM con un rango dinámico de 0 a 100\%. Además, se cumple que el torque y el momento son proporcionales a dicha señal de comandos. De esta forma se obtienen las ecuaciones (7), (8) y (9).

$$
\begin{aligned}
& U_{1}=b\left(y_{4}-y_{2}\right) \\
& U_{2}=b\left(y_{3}-y_{1}\right) \\
& U_{3}=d\left(y_{2}+y_{4}-y_{1}-y_{3}\right)
\end{aligned}
$$




\begin{tabular}{|c|c|c|}
\hline Símbolo & Definición & Unidad \\
\hline$\phi$ & Roll & $\mathrm{rad}$ \\
\hline$\theta$ & Pitch & $\mathrm{rad}$ \\
\hline$\psi$ & Yaw & $\mathrm{rad}$ \\
\hline$\Omega_{i}$ & $\begin{array}{l}\text { Velocidad del } \\
\text { rotor }\end{array}$ & $\frac{\mathrm{rad}}{\mathrm{seg}}$ \\
\hline$I_{x, y, z}$ & $\begin{array}{l}\text { Inercia del } \\
\text { cuerpo }\end{array}$ & $K g r m^{2}$ \\
\hline$b$ & Factor de empuje & $\frac{N}{\%}$ \\
\hline$d$ & Factor de torque & $\frac{N m}{\%}$ \\
\hline$l$ & Palanca & $m$ \\
\hline$b^{\prime}$ & $\begin{array}{l}\text { Factor de empuje } \\
\text { para velocidad }\end{array}$ & $\frac{N}{(r)^{2}}$ \\
\hline$d^{\prime}$ & $\begin{array}{l}\text { Factor de torque } \\
\text { para velocidad }\end{array}$ & $\frac{N m}{\left(\frac{r}{-2}\right)^{2}}$ \\
\hline$y_{1,2,3,4}$ & $\begin{array}{l}\text { Comandos a } \\
\text { actuadores }\end{array}$ & $\%$ \\
\hline
\end{tabular}

Tabla 1 - Símbolos y definiciones. 


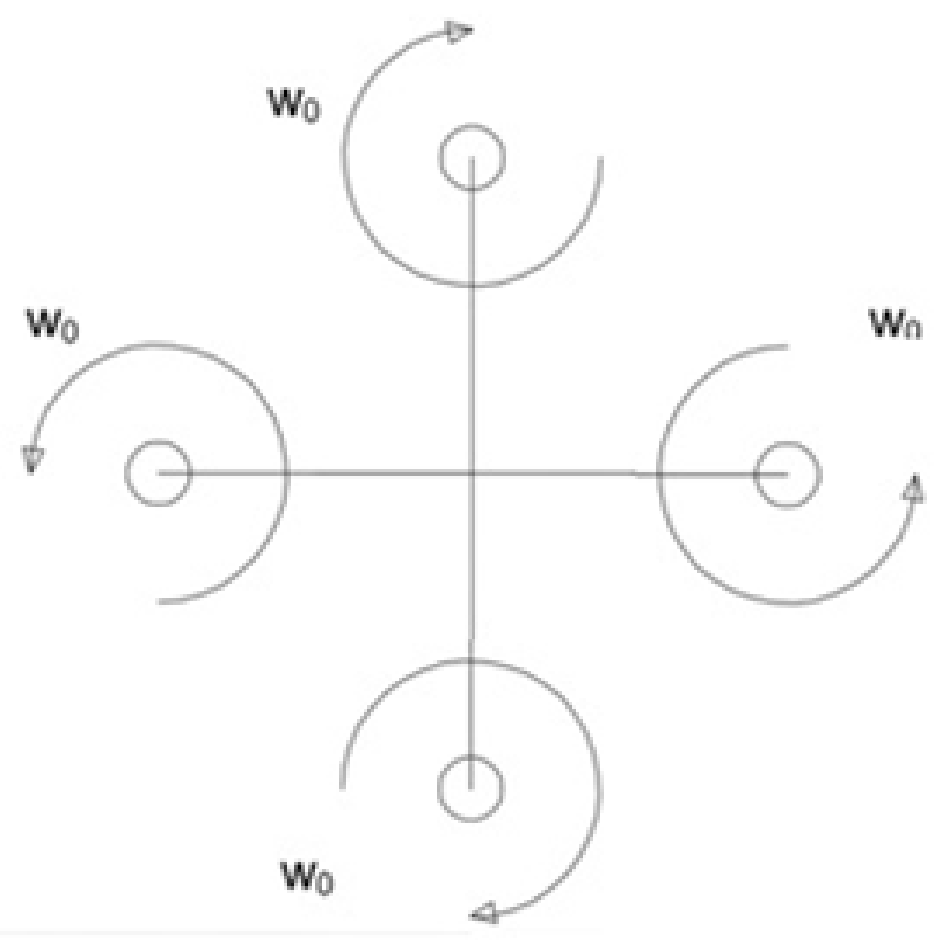

Fig. 1 - Sentido de giro de los actuadores.
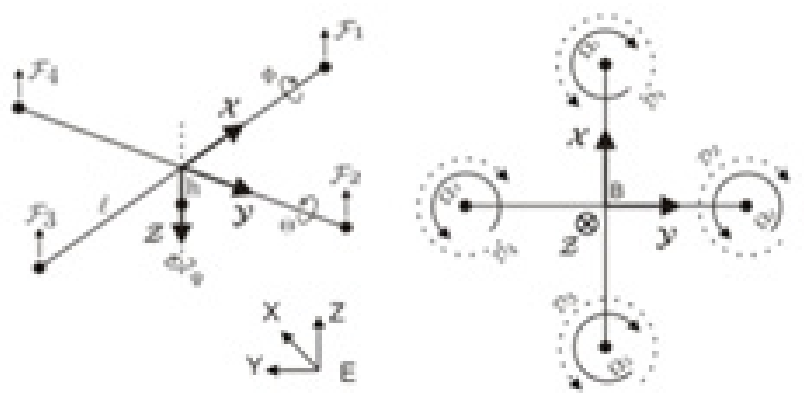

Fig. 2 - Terna de ejes adoptada (Bouabdallah et al., 2007).

Dentro de los parámetros a cuantificar podemos hallar dos grupos: los parámetros de los actuadores y los parámetros de la planta. Los primeros son el factor de empuje y el factor de torque, y los segundos son los momentos de inercia en cada eje de rotación del cuerpo del drone. También, se realizarán los ensayos 
correspondientes para determinar que la hipótesis de tener un amortiguamiento despreciable en los actuadores es cierta desde un punto de vista práctico para nuestro caso de estudio.

\section{Parámetros a cuantificar}

Los parámetros que medimos ensayando a los actuadores son la curva de empuje-potencia, la curva de torque-potencia, la curva de velocidad-potencia y el tiempo de respuesta que tarda el motor en alcanzar una nueva velocidad (Kharsansky, 2013) (Tavares, 2014). La potencia es indicada como porcentaje de exigencia, que es el modo en que la computadora de control se comunica con el controlador ESC, mediante señal de PWM.

Los parámetros medidos de la planta son el brazo de palanca y la inercia. El brazo de palanca es la distancia entre el actuador y el centro de gravedad del vehículo. La inercia se conforma por la masa, que influye en las traslaciones, y el tensor de inercia, que es una matriz de 3x3 que influye en los movimientos de rotación. Se obtienen sus valores mediante ensayo, aunque también pueden ser calculados mediante programas de CAD u obtenerse un valor aproximado mediante simplificaciones. La planta se supone un cuerpo perfectamente rígido en todos los cálculos.

En la tabla 2 se resumen los parámetros a cuantificar.

\begin{tabular}{|c|l|c|}
\hline Símbolo & Definición & Unidad \\
\hline$l$ & Palanca & $\mathrm{m}$ \\
\hline$b$ & $\begin{array}{l}\text { Factor de } \\
\text { empuje }\end{array}$ & $\frac{\mathrm{N}}{\%}$ \\
\hline$d$ & $\begin{array}{l}\text { Factor de } \\
\text { torque }\end{array}$ & $\frac{\mathrm{Nm}}{\%}$ \\
\hline$I_{x, y, z}$ & $\begin{array}{l}\text { Inercia del } \\
\text { cuerpo }\end{array}$ & $\mathrm{Kgr} \mathrm{\textrm {m } ^ { 2 }}$ \\
\hline
\end{tabular}

Tabla 2 - Parámetros a cuantificar

El diseño y la fabricación de los bancos de ensayo fueron realizados en nuestro laboratorio, contando con una impresora 3D.

Para realizar los ensayos, se utilizaron 4 motores brushless A2212 1000KV, 4 controladores de velocidad electrónicos (ESC) modelo XXDHW30A, 4 hélices de 10x4.5" y una placa de desarrollo STM32F4 Discovery. 


\section{Ensayos de empuje (thrust) y torque (drag)}

Para el ensayo de las fuerzas generadas por el actuador, se utiliza un instrumento de medición de fuerza y un banco capaz de transferir al mismo el empuje y el torque generado, a lo largo de una progresión de potencia.

Posteriormente, se modelizan los motores siguiendo un modelo único, que representa a cada uno con aceptable precisión. El modelo se simplifica a una función lineal, tangente a la curva obtenida, en el punto de la función donde el empuje es el necesario para mantener la posición de hovering.

\section{Banco de ensayo}

El modelo de banco utilizado consiste en un efecto palanca de relación 2:1 para el empuje y un largo de $100 \mathrm{~mm}$, que se debe considerar en la medición de torque, ya que el empuje soportado por el instrumento es función del torque y del largo del brazo. Como instrumento de medición hemos utilizado una balanza digital. Otra opción es utilizar un instrumento de mayor respuesta que registre los valores para obtener una medición más real y luego filtrar los ruidos de la medición utilizando un software adecuado.

Se ha elegido este modelo respecto a otros ya que es el que, con mayor facilidad, permite la medición tanto de empuje como de torque, debiendo solo desmontarse el motor y montarse luego con una diferencia de $90^{\circ}$.

Se debe tener en cuenta en el diseño que la posición de la palanca se encuentre de forma horizontal y la medición obtenida no posea errores por una alineación inadecuada.

Asimismo, debe haber una zona libre por debajo del actuador de al menos un diámetro de la hélice, de modo que el viento generado no colisione y nos permita realizar la medición sin el llamado 'efecto suelo'. El efecto suelo se genera cuando la distancia entre la hélice y el suelo es menor al diámetro de la misma, generando un desalojo del aire menor, lo que eleva la presión debajo de la hélice y genera que el empuje del motor aumente.

La fuerza generada por el motor para cada muestra se muestran en las ecuaciones (9) y (10) (Fliess, 1970).

$$
B=F * r
$$

Donde F: fuerza de empuje del actuador; r: relación del banco de ensayo (2:1); B: valor obtenido en el instrumento de medición. 
Mientras que la medición del torque, se calcula con la ecuación (10).

$$
T=B * L
$$

Donde T: Torque del actuador; L: Distancia horizontal desde el punto de giro del brazo hasta el instrumento de medición $(100 \mathrm{~mm})$.

\section{Modelos alternativos}

Un modelo alternativo consiste en construir una estructura donde el empuje se encuentra alineado directamente hacia el instrumento de medición. Puede fabricarse de crecimiento negativo (figura 3) o positivo (figura 4), en el primer caso se utiliza un peso muerto inicial mayor al empuje máximo que puede generar el conjunto motor-hélice ensayado. En el modelo de crecimiento positivo, la medición es directa. En el modelo de crecimiento negativo, el instrumento de medición comienza desde un valor máximo igual al peso muerto y a medida que se aumenta la potencia, se calcula el valor de empuje por una simple resta. Este modelo es utilizable sólo para el empuje y debe ser construido de modo que pueda soportar el torque sobre el estator.

Otros modelos de banco con efecto de palanca poseen doble brazo, ubicados de forma paralela, que evitan los problemas de alineación para el ensayo de empuje (figura 5). Para el ensayo de torque, de todos modos, la alineación sí debe ser correctamente horizontal.

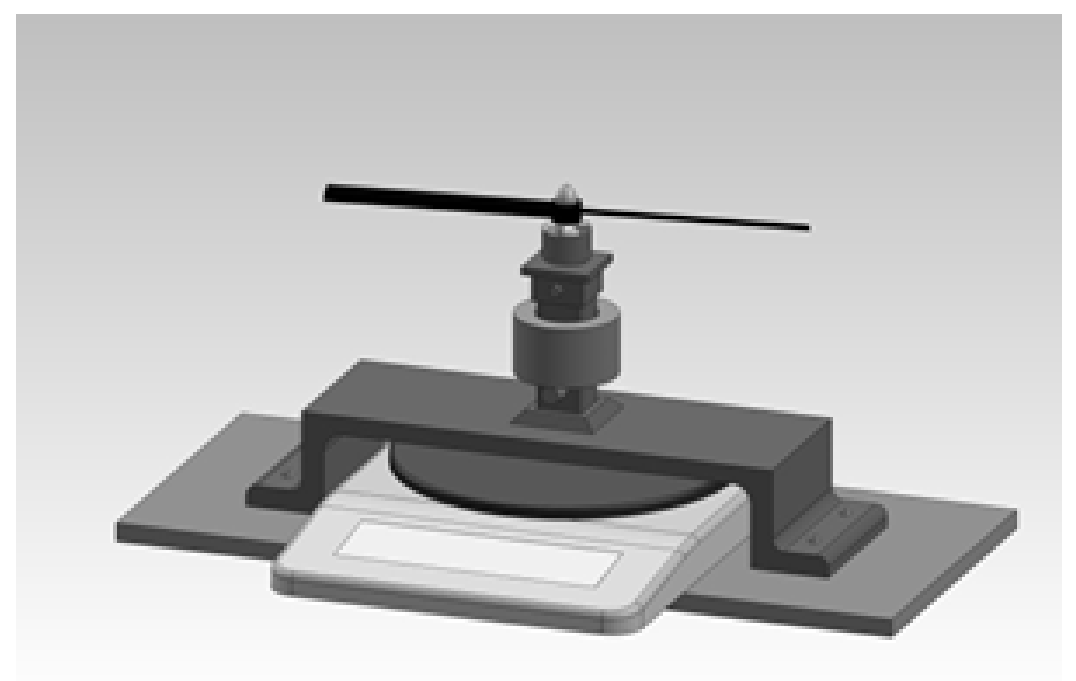

Fig.3 - Medición de empuje con crecimiento negativo. 


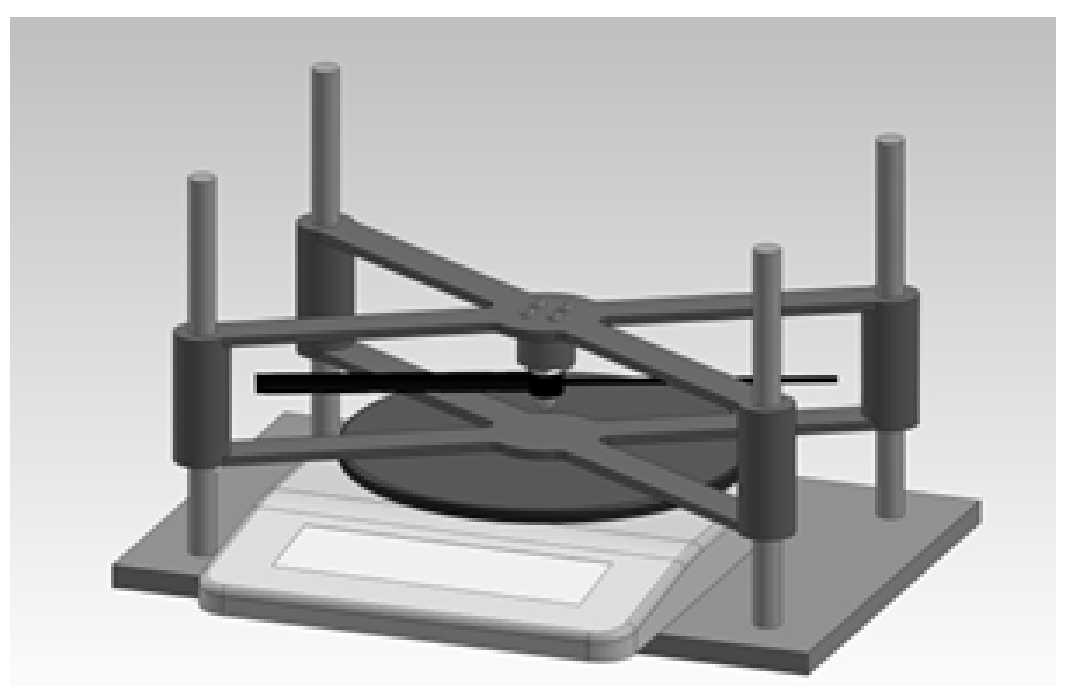

Fig. 4 - Medición de empuje con crecimiento positivo.

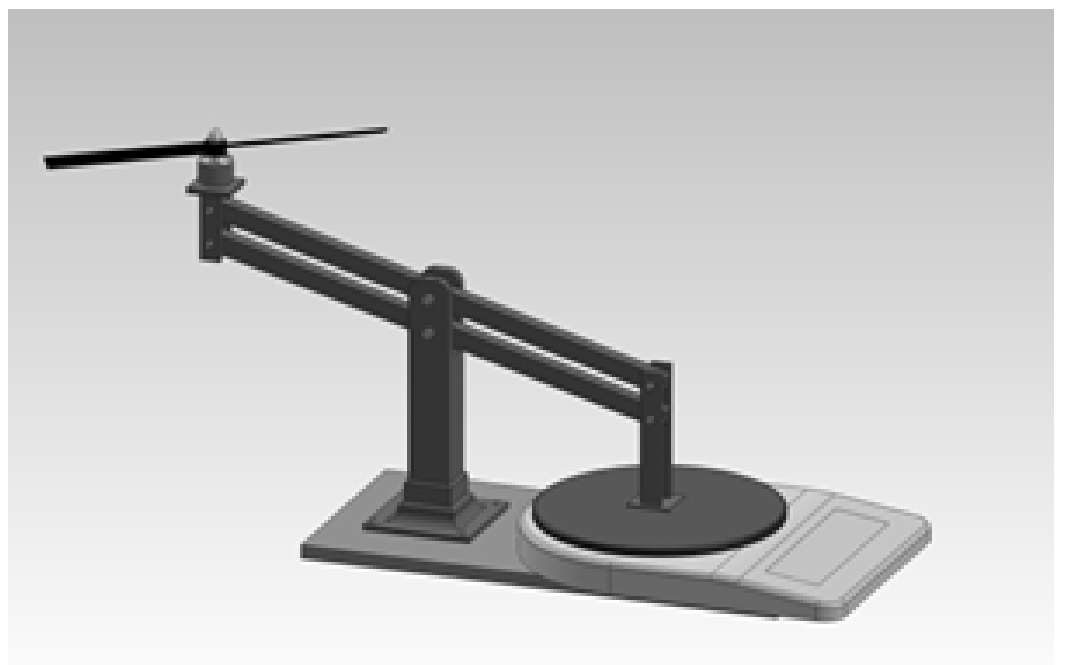

Fig. 5 - Medición de empuje con banco de ensayo de doble brazo.

\section{Realización del ensayo}

Se dispuso el banco sujetándolo con pinzas y llave de fuerza a una mesa o superficie lo suficientemente rígida. Se colocó el motor atornillándolo al extremo correspondiente y por debajo del opuesto se colocó una balanza digital PRECision 
Serie TH. Se realizaron las conexiones eléctricas pertinentes, incluyendo el ESC, batería y la placa controladora y de adquisición.

Se alimenta al motor para obtener una potencia del $0 \%$ inicialmente hasta una potencia del $100 \%$, tomando arbitrariamente mediciones cada un intervalo de $2 \%$, de modo que obtendremos un muestreo con 51 puntos. De esta manera se obtiene una curva de porcentaje de potencia-empuje (thrust) y una de porcentaje de potencia-torque (drag) por cada motor.

\section{Resultados de la medición de empuje}

En la figura 6 se observa la relación medida entre el ciclo de actividad (duty) del PWM y el empuje generado por el actuador.

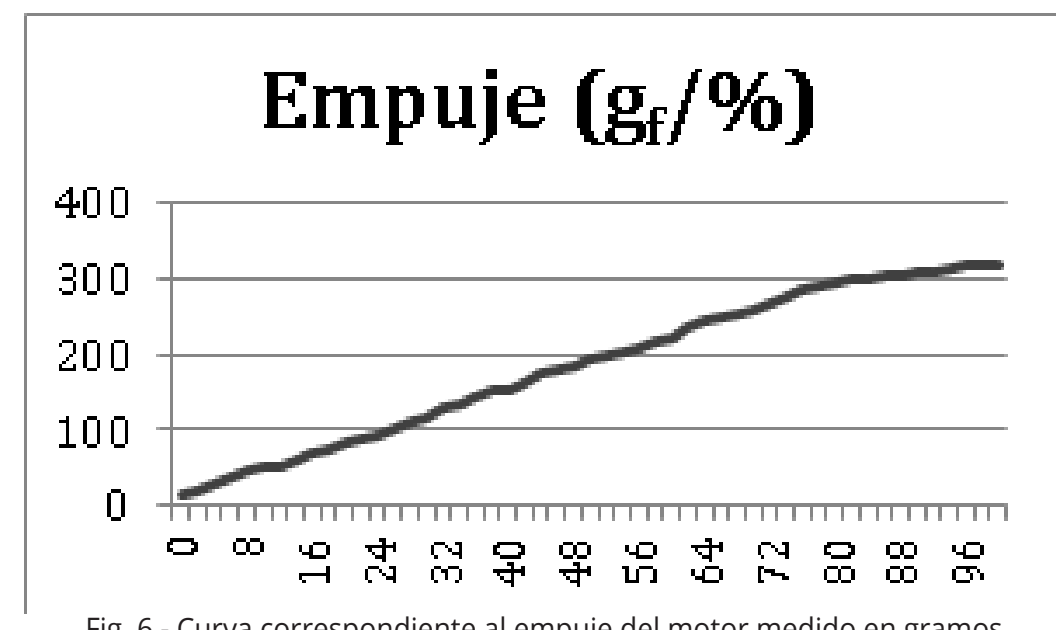

Fig. 6 - Curva correspondiente al empuje del motor medido en gramos fuerza, para cada entrada de PWM enviado al ESC.

Se linealiza la curva en base a la tangente en el punto de trabajo, correspondiente al peso del drone dividido la cantidad de motores, ya que es el empuje que debe realizar cada motor en hovering.

El peso de la planta utilizado es de 793 gramos y como ejemplo se linealiza para 4 motores, de modo que el punto de trabajo será el valor de 198,25 gramos fuerza, que corresponde al 51\%. Los valores disponibles de la medición son 194,5 gramos fuerza a $50 \%$ y 200 gramos fuerza a $52 \%$, por lo tanto, la pendiente tendrá un valor de 2,75 gramos fuerza por cada porcentaje de ciclo de actividad.

En consecuencia, nos queda la ecuación final (11). 


$$
F=2,75 \frac{g_{f}}{\%} * \delta * 100 \%+57 g_{f}
$$

Si pasamos a newton nos queda la ecuación (12).

$$
F=0,0275 \frac{N}{\%} * \delta * 100 \%+0,57 N
$$

Siendo $\delta$ el ciclo de actividad expresado entre 0 y 1 .

\section{Resultados de la medición de torque}

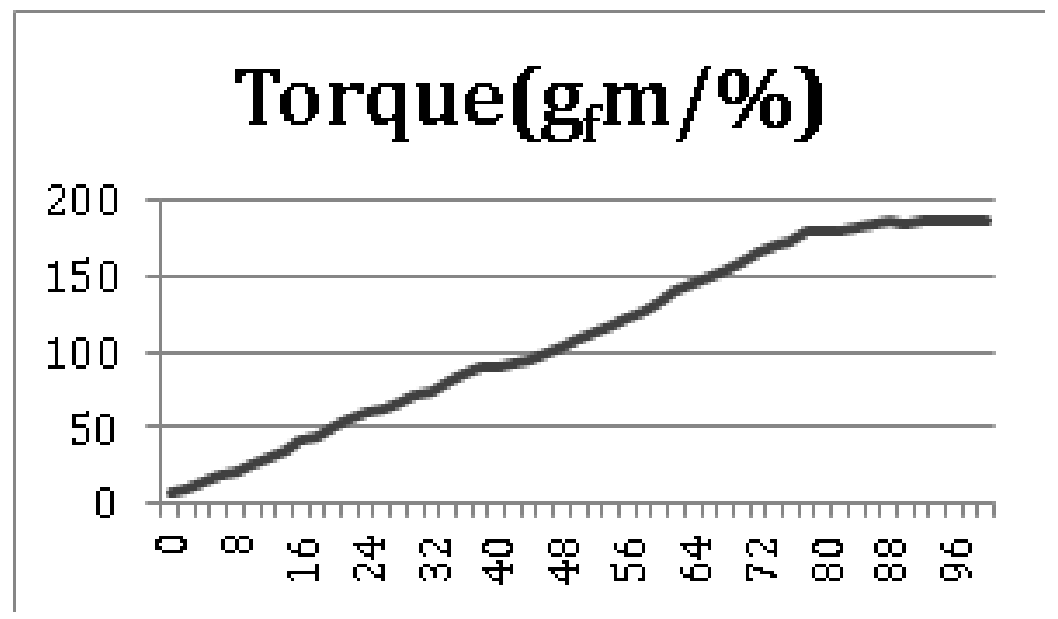

Fig. 7 - Curva correspondiente al torque del motor medido en gramos fuerza por metro, para cada entrada de PWM enviado al ESC.

En la figura 7 se observa la relación medida entre el ciclo de actividad del PWM y el empuje generado por el actuador. Se linealiza mediante la tangente en el mismo punto de trabajo que la curva de empuje, que resultó en 51\%. Éste corresponde a un torque generado de 110,5 gramos fuerza por metro y una pendiente de 1,5 gramos fuerza por metro por cada porcentaje de ciclo de actividad.

En consecuencia, nos queda la ecuación (13). 


$$
M=1,5 \frac{g_{f} m}{\%} * \delta * 100 \%+34 g_{f} m
$$

Si pasamos a newton-metro nos queda ecuación (14).

$$
M=0,015 \frac{\mathrm{Nm}}{\%} * \delta * 100 \%+0,34 \mathrm{Nm}
$$

Finalmente, podemos decir que el factor de empuje vale $0.027 \mathrm{~N} / \%$, y el factor de torque vale $0.015 \mathrm{Nm} / \%$ para el punto de trabajo considerado. Ambos, corresponden a los parámetros b y d de la tabla 2 respectivamente.

\section{Medición del brazo de palanca (lever)}

En este trabajo se utilizó un chasis (frame) comercial DJI F450 donde el brazo es de $0.225 \mathrm{~m}$, con lo cual tenemos la constante 1 de la tabla 2.

\section{Ensayos de momentos de inercia}

Conocer los momentos de inercia de la planta es tan importante como conocer las constantes características de los actuadores. Ésta es la otra magnitud física fundamental que afecta en la dinámica del drone. Los momentos de inercia de un cuerpo rígido pueden representarse en una matriz de $3 \times 3$, conocida como Tensor de inercia, asociada a 3 ejes perpendiculares. Se trata de una matriz simétrica, cuyos valores de la diagonal representan los momentos de inercia I_x, I_y e I_z(también llamados I_xx, I_yy e I_zz), en donde cada valor está asociado a un eje. Los demás valores de la matriz son los llamados momentos centrífugos, cuyos valores están asociados a 2 ejes cada uno (I_xy=I_yx; I_xz=I_zx;I_yz=I_zy) (Beer et al., 1997) (Beer et al., 1998).

Para todo cuerpo rígido existe al menos una terna de ejes perpendiculares entre sí, cuyos momentos centrífugos son nulos. Estos ejes reciben el nombre de ejes principales de inercia, y poseen la particularidad de estar asociados a un tensor de inercia diagonal.

En un cuerpo rígido que posee dos planos de simetría perpendiculares entre sí, se puede asegurar que éstos y el plano perpendicular a ambos forman la terna de ejes principales de inercia. Como es el caso de nuestra estructura, para cualquier cuadricóptero, podemos asegurar que la terna de referencia será una terna de ejes principales de inercia. 
De este modo, conocer los momentos de inercia I_x,I_y e I_z es suficiente para conocer el tensor de inercia de la planta.

\section{Banco de ensayo}

Cada momento de inercia puede ser calculado midiendo la respuesta cinemática del cuerpo sólido en un ensayo de péndulo bifilar.

Un péndulo bifilar consiste en suspender al drone de dos fibras paralelas y a igual distancia del centro de gravedad del drone. El eje al cual se encuentra asociado el momento de inercia a medir debe quedar orientado paralelo a la dirección de la gravedad.

Debe considerarse que el largo de las fibras (L) debe ser considerablemente mayor a la distancia entre el centro de masa y el punto de sujeción (R). Además, el ángulo inicial de desplazamiento debe ser pequeño, ya que para el desarrollo matemática se utiliza la simplificación $\sin (\phi) \approx \phi$, la cual sólo es válida para ángulos pequeños.

\section{Realización del ensayo}

Para comenzar el ensayo, se da un ángulo inicial de desplazamiento al drone, respecto de la dirección en la que se desea medir, y se deja oscilar una cantidad de veces definida. A menor cantidad de oscilaciones, mayor error de medición en el tiempo de una oscilación. A mayor cantidad de oscilaciones, mayor error al despreciar el trabajo de fuerzas no conservativas, como ser la viscosidad del aire, o el rozamiento en las fibras. En nuestro ensayo, utilizamos 10 oscilaciones.

Una vez conocido el tiempo de una oscilación (T), llamado período, se procede a aplicar la fórmula (15) (Schermuk, 2012).

$$
I=\frac{1}{4 \pi^{2}} * \frac{M * g * R^{2} * T^{2}}{L}
$$

Siendo M la masa y g la gravedad.

El ensayo se repite por cada eje, siendo un total de 3 ensayos (figura 8). 


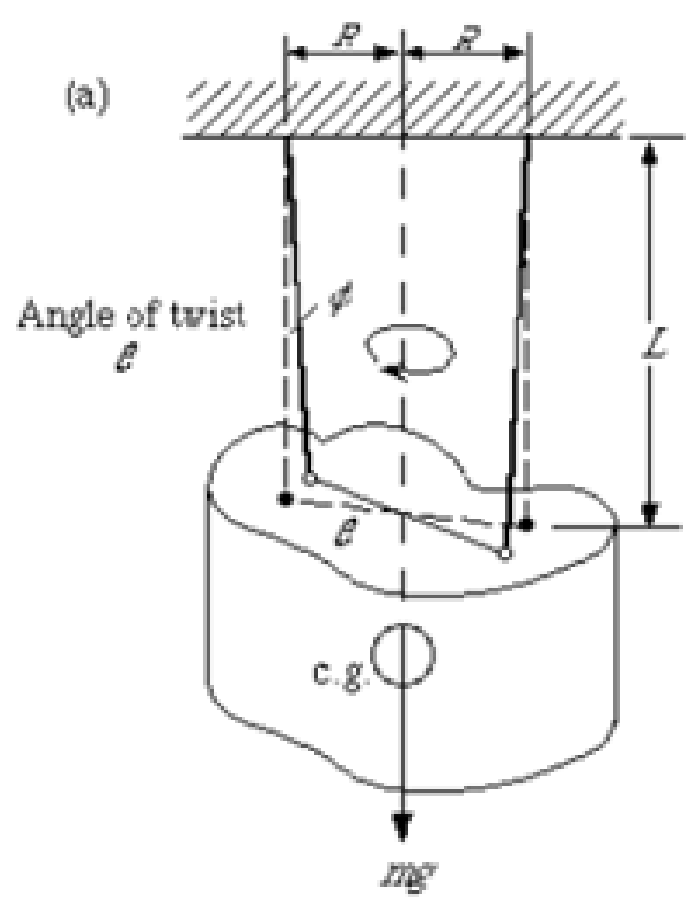

Fig. 8 - Experimento del péndulo bifilar para obtener el tensor de inercia del drone (Kharsansky, 2013).

Finalmente, en la ecuación (16) se muestra el resultado obtenido para el parámetro I_(x,y,z) de la tabla 2.

$$
I_{x, y, z}=\left[\begin{array}{ccc}
0.0081 & 0 & 0 \\
0 & 0.0092 & 0 \\
0 & 0 & 0.0198
\end{array}\right] \mathrm{Kgm}^{2}(16)
$$

\section{Ensayo de velocidad de giro y respuesta}

El ensayo de velocidad de giro de un actuador es un ensayo complementario que se utiliza para conocer la curva empuje-velocidad de giro y torque-velocidad de giro de un aspa, y en controles más complejos se utiliza para conocer las fuerzas adicionales que aparecen cuando el UAV vuela con una velocidad relativa respecto del aire o para conocer la magnitud de los efectos giroscópicos (producidos cuando el eje de giro de un cuerpo cambia de dirección mientras éste posee una velocidad angular). En nuestro ensayo, obtuvimos la curva porcentaje de potencia-velocidad 
de giro.

Como el actuador posee un momento de inercia propio, la velocidad de giro no puede modificarse de forma instantánea. Por eso, existe una diferencia entre la velocidad que el sistema de control envía al motor, y la velocidad con la que éste gira, hasta alcanzar la velocidad requerida. Sin embargo, como el tiempo de respuesta del motor es lo suficientemente elevado como para no provocar una diferencia significante en la posición del drone, se supone una respuesta instantánea. El ensayo de respuesta al escalón se realiza para confirmar la validez de esta suposición.

\section{Banco de ensayo}

Para este ensayo sólo se requiere de un banco capaz de sujetar al actuador en todo momento. En las figuras 9 y 10 se ilustra el banco propuesto.

Esta medición puede realizarse directamente sobre el mismo banco que se utilizó para las mediciones de cargas. En nuestro caso, utilizamos una base metálica con peso suficiente para permanecer inmóvil con el motor a máxima carga y una columna, en cuyo extremo se monta el motor, con altura mayor a un diámetro de aspa, para recrear las condiciones similares al vuelo libre, ya que una altura menor provoca el 'efecto suelo', mencionado anteriormente.

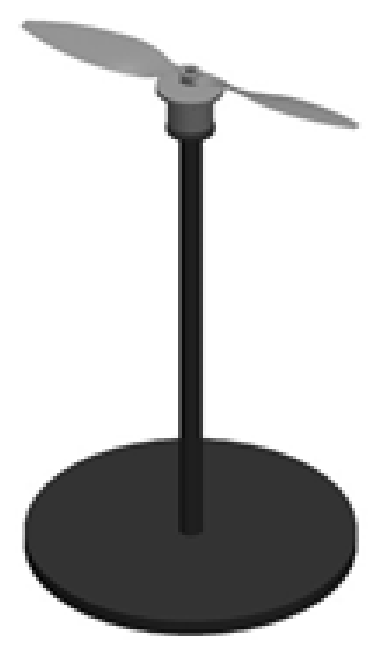

Fig. 9 - Vista del banco para ensayo de velocidad de giro. 


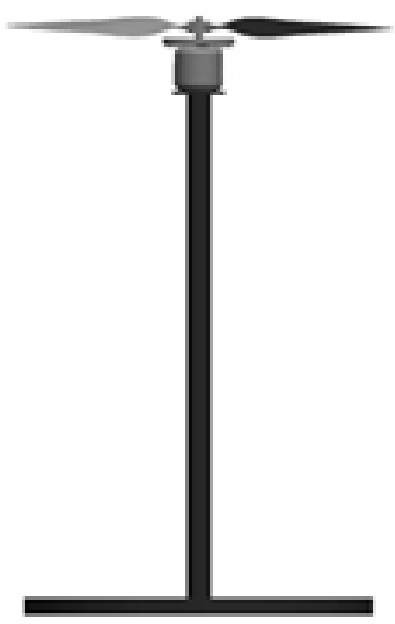

Fig. 10 - Perfil del banco de ensayo de velocidad de giro.

\section{Método de medición}

Con el objetivo de obtener la frecuencia a la cual gira el motor en cada instante, en primera instancia se evalúa la señal proveniente de una de sus fases con su respuesta en frecuencia, tal como se puede observar en la figura 11. La misma fue tomada mediante un osciloscopio para la frecuencia máxima de giro del motor utilizado, esto es, enviando al ESC un PWM de 100\% de ciclo de actividad.
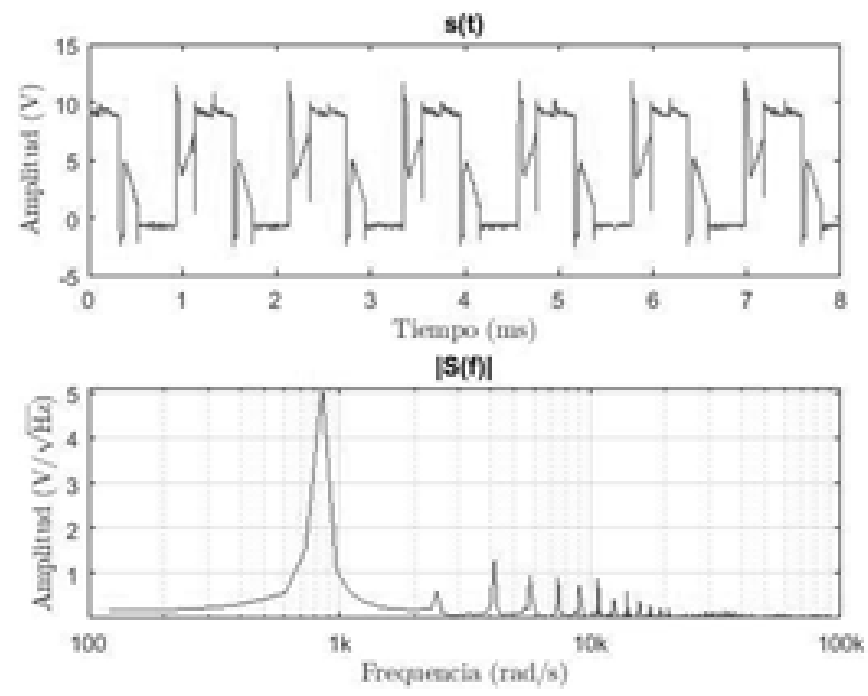

Fig. 11 -Señal proveniente de una fase del motor y su respuesta en frecuencia. 
En base a esto, para evitar los sobrepicos presentes en la señal medida, se propone realizar un filtro pasabajos que suavice dicha señal, además de un filtro pasa altos para eliminar la continua. El Diagrama de Bode del filtro propuesto puede observarse en la figura 12.

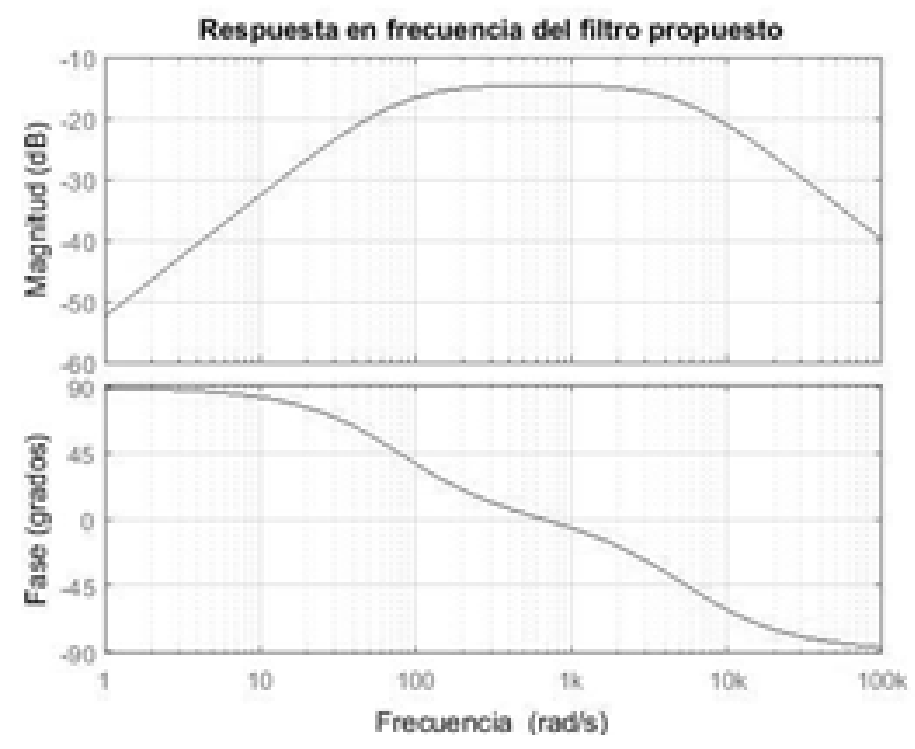

Fig. 12 - Respuesta en frecuencia del filtro propuesto

La señal filtrada se asemeja a una senoidal, por lo que mediante el uso de un amplificador operacional se pueden obtener pulsos proporcionales a la señal propia del motor. El circuito puede observarse en la figura 13, donde como primer etapa se encuentra el filtro desarrollado anteriormente, y luego mediante la comparación de la señal montada a una continua y esa misma continua genera los pulsos que irán a un pin digital de la placa de medición. Se monta en continua la señal ya que se utilizó un amplificador alimentado con fuente simple, haciendo imposible la detección de la señal por debajo de los $0 \mathrm{~V}$. 


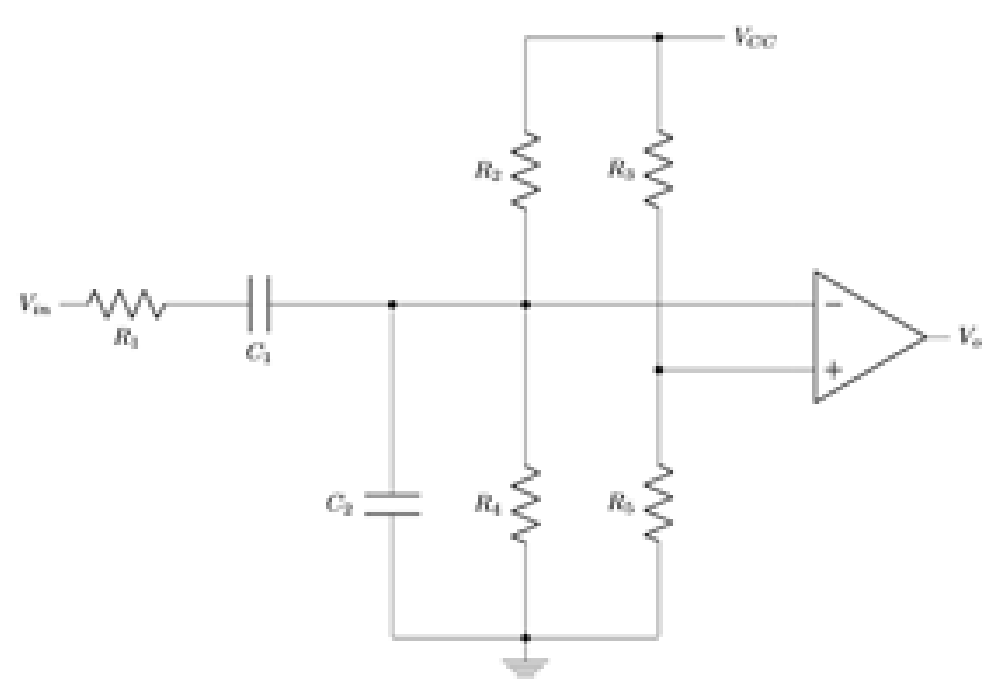

Fig. 13 -Circuito propuesto para la medición de frecuencia.

Finalmente, en la figura 14 puede observarse la relación entre el pulso generado y el período de la señal proveniente del motor.
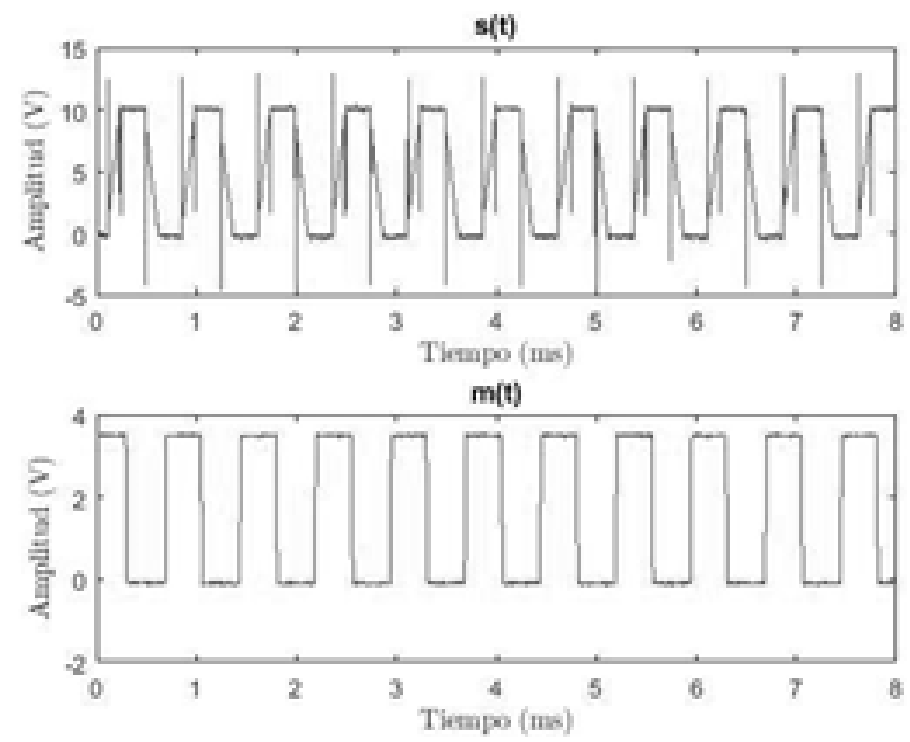

Fig. 14 - Señal proveniente del motor $(s(t))$ y señal a la salida del circuito $(m(t))$.

\section{Realización del ensayo}

Se ajustó el actuador sobre el banco, se conectó la placa de medición y la fuente 
de alimentación. En consecuencia, se procedió a realizar un barrido de potencia, desde $0 \%$ hasta $100 \%$, cada un intervalo de $2 \%$, obteniendo nuevamente 51 puntos de medición, correspondientes a los mismos valores de entrada que para los ensayos de carga.

Debido al principio de medición, lo valores obtenidos corresponden a frecuencias eléctricas del motor, las cuales tiene su correlato con la frecuencia mecánica (Hanselman, 2006) mediante la ecuación (17).

$$
f_{\text {mecánica }}=\frac{N_{\text {polos }}}{f_{\text {eléctrica }}}
$$

El número de polos corresponde al número de polos magnéticos del motor y se obtiene de las especificaciones técnicas. En nuestro caso N_polos=14 (Rhydolabz, 2018).

El ensayo se realiza para cada motor y se linealiza un único modelo, el cual puede observarse en la figura 15, en donde se llevaron los valores a rpm.

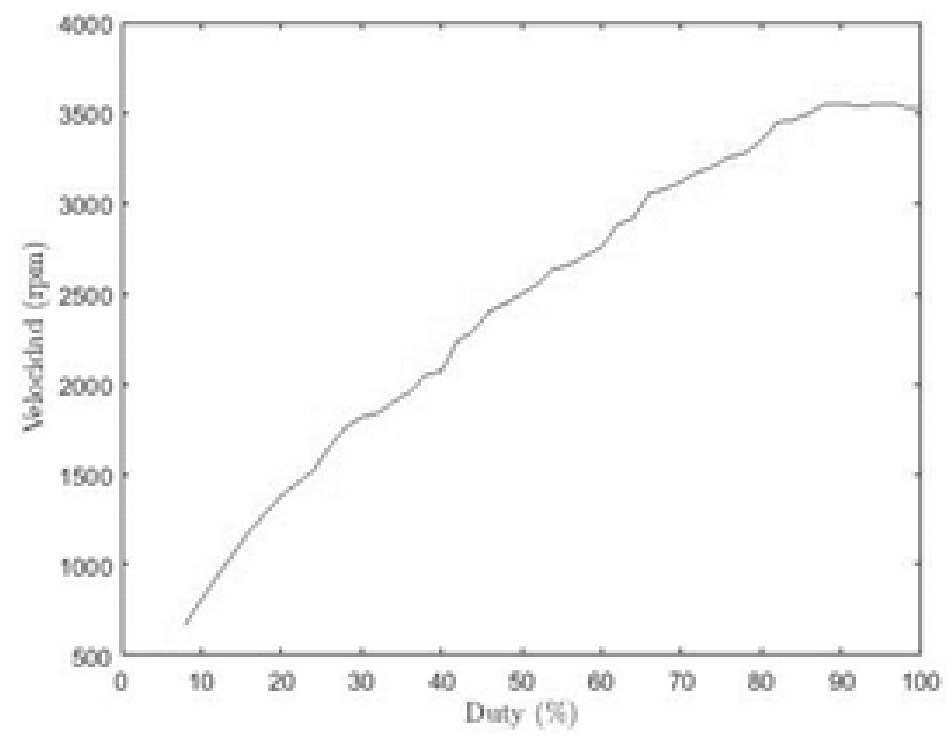

Fig. 15 - Velocidad en frecuencia del sistema en base al ciclo de actividad.

Al igual que con las curvas de torque y empuje, se linealiza la misma en el punto de trabajo, para el valor de 51\%, que corresponde a $2513 \mathrm{rpm}$. 
La pendiente para dicho valor corresponde a $32,87 \mathrm{rpm}$ por cada porcentaje de ciclo de actividad. En consecuencia, se obtiene la ecuación (18), donde el ciclo de actividad $\delta$ corresponde a su valor entre 0 y 1 y n la velocidad en revoluciones por minuto.

$$
n=32,87 \mathrm{rpm} * \delta * 100+836,65 \mathrm{rpm}
$$

En la figura 16 puede observarse la velocidad de respuesta del motor en el punto de trabajo en base a la señal de excitación con saltos de $4 \%$ de ciclo de actividad.

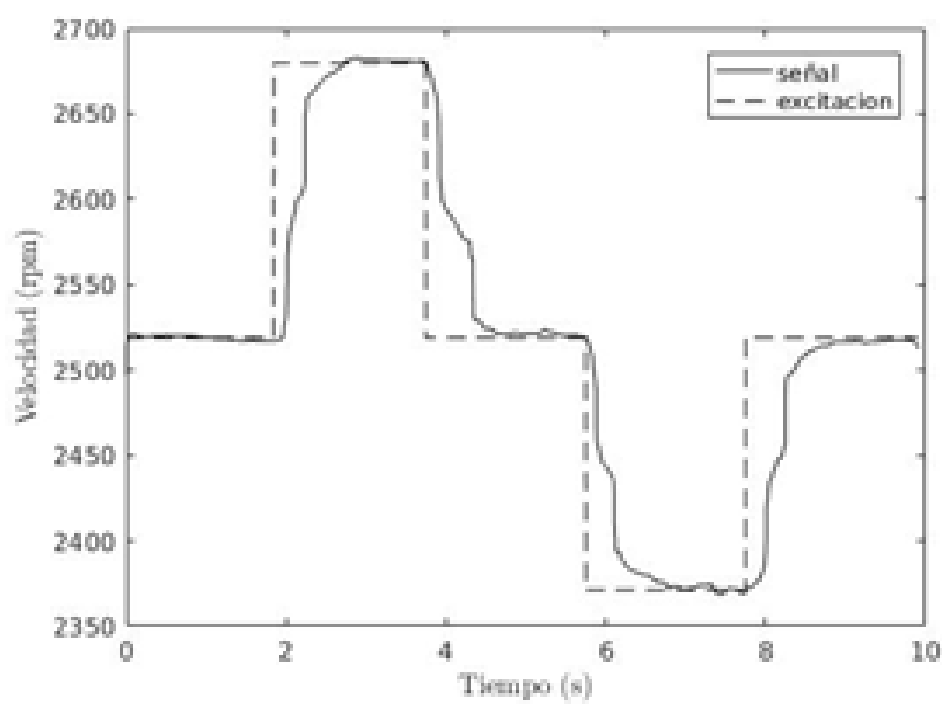

Fig. 16: velocidad de respuesta del actuador respecto a un escalón.

Se observa a partir de dicha figura que la demora del actuador en alcanzar el valor requerido es aproximadamente de 400 milisegundos para el aumento de ciclo de actividad y 500 milisegundos para la disminución de dicho ciclo de actividad. Dicho valor puede ser considerado para calcular la acción de control del sistema o despreciarse aceptando el error que genera.

\section{Ensayo de estabilización}

El ensayo de estabilización es el ensayo final antes del vuelo libre, donde se puede verificar, con un solo grado de libertad, el correcto funcionamiento del sistema de control electrónico del UAV. Se tiene un ensayo para pitch y roll, y otro para yaw.

Para ensayar la estabilización en pitch y roll se monta la nave lista para volar, 
con sólo dos actuadores conectados, en una estructura que la mantiene firme y permite sólo su rotación sobre un eje. El UAV debería ser capaz de mantener la posición paralela al suelo, y recuperar esa posición de manera eficiente ante la presencia de una perturbación.

Para ensayar la estabilización en yaw se monta la nave lista para volar con los cuatro actuadores conectados a una estructura con un sistema tal que permita solo la rotación sobre el eje vertical.

Al realizar estos ensayos, se pone en juego, en primer lugar, el modelo de control, y en segundo lugar, las constantes calculadas en los ensayos anteriores.

\section{Banco de ensayo para pitch y roll}

El banco sujeta dos brazos del vehículo con dos piezas plásticas, montadas sobre rodamientos alineados, de modo que el eje de los rodamientos es el eje de movimiento del drone. Los brazos no sujetados son aquellos que llevan las aspas colocadas y que el drone utiliza para estabilizarse (figura 17 y 18).

El banco utilizado es de construcción sencilla y es importante destacar que, si bien el eje coincide con la dirección de dos de los brazos, el método sirve tanto para un modelo de control diseñado para vuelo en '+' o ' $x$ ', y no sólo con 4 actuadores. La única diferencia es el modo en que el sistema de control interpreta la medición de los ángulos.

Para realizar el ensayo de estabilidad en pitch y roll se implementó un sistema de control sobre una computadora electrónica basada en un microcontrolador STM32F 4 con un procesador ARM Cortex-M4. Para medir instante a instante las posiciones y velocidades angulares se utilizó una unidad de medición inercial MPU9250. Para la actuación sobre la planta se utilizaron los actuadores caracterizados a lo largo del trabajo. Los datos obtenidos por telemetría mostraron que el desempeño del control de estabilidad del cuadricóptero fue satisfactorio. Si bien el diseño y desarrollo del sistema de control están fuera del alcance del presente trabajo, cabe aclarar que se obtuvieron valores de pitch y roll dentro de un rango de $\pm 4^{\circ}$ con media en $0^{\circ}$ (condición de hovering). 


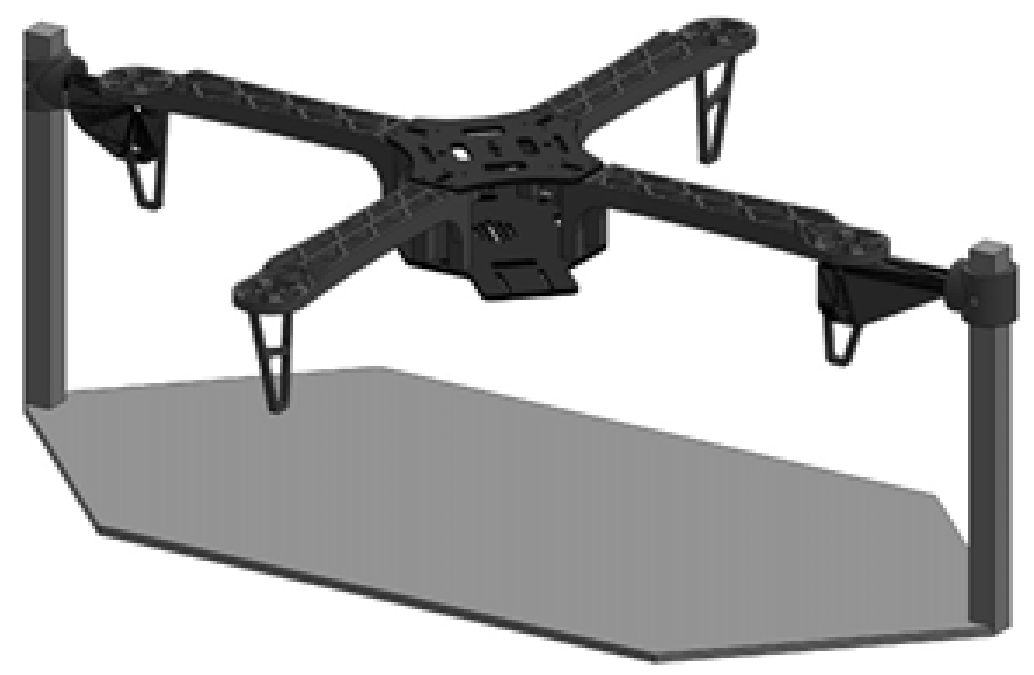

Fig. 17: vista del banco de ensayo de estabilización en pitch y roll.

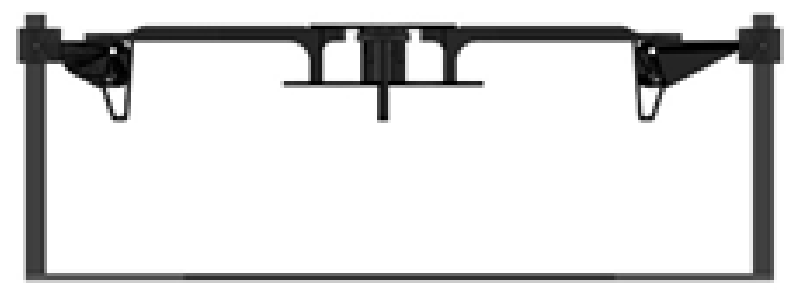

Fig. 18: perfil del banco de ensayo de estabilización en pitch y roll.

\section{Banco de ensayo yaw}

Se emplea el principio de articulación rotacional. Suministra un grado de libertad consistente en una rotación alrededor del eje de la articulación.

El banco es de construcción sencilla, consiste en una varilla que posee una base para ser amurada al suelo o a un banco de mayor rigidez, y en el otro extremo posee un rodamiento con una base incorporada para sujetar al drone y permitirle rotar sobre su eje vertical (figura 19 y 20). Si bien se llevó a cabo el diseño del banco, no se realizaron ensayos de yaw para este trabajo. 


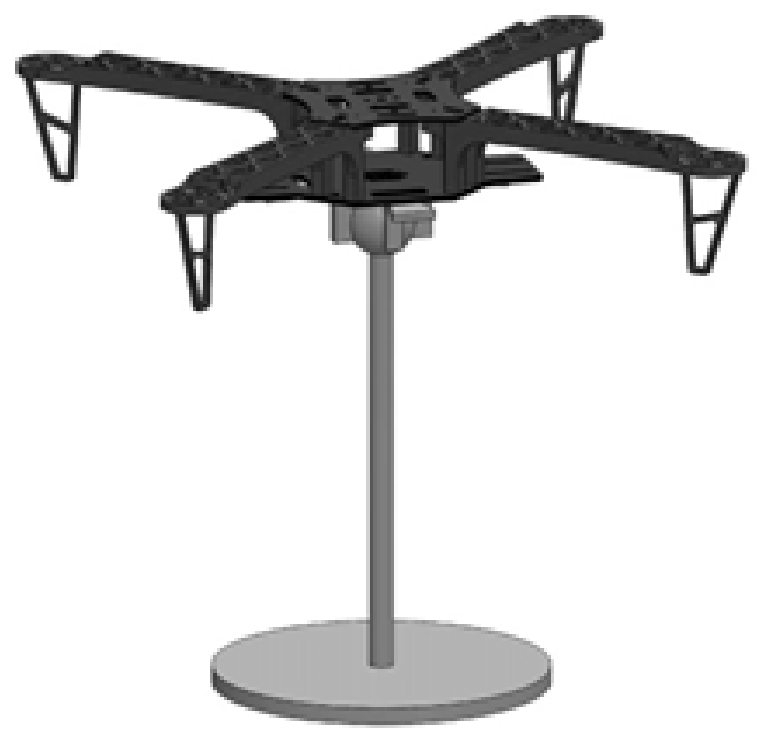

Fig. 19: vista del banco de ensayo de estabilización en los tres grados de libertad de rotación.

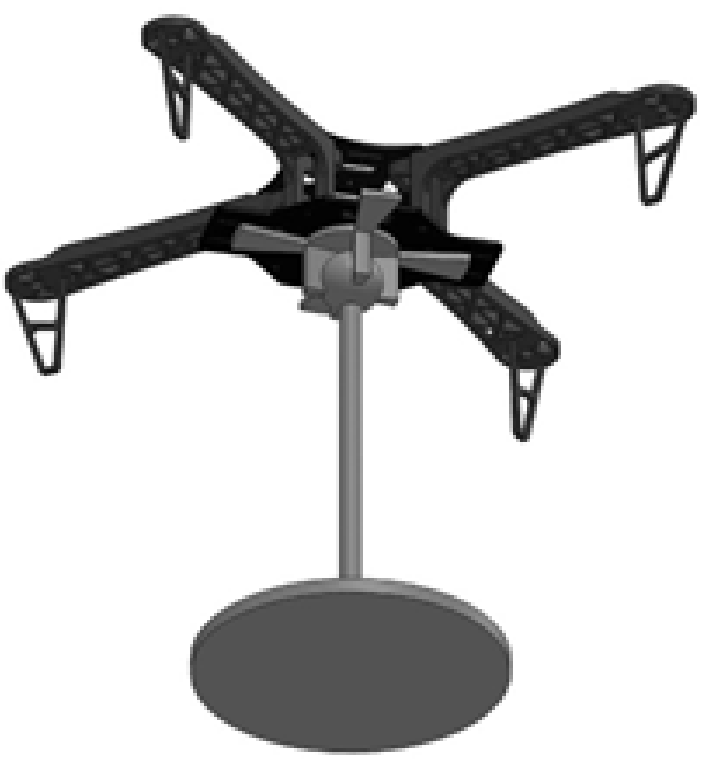

Fig. 20: vista desde abajo del banco de ensayo de estabilización en yaw. 


\section{CONCLUSIONES}

En este trabajo se presentaron los ensayos necesarios para la caracterización de los parámetros de un drone con formato cuadricóptero. Con esta caracterización, es posible el diseño y un ajuste inicial de los sistemas de control electrónicos a bordo de la aeronave.

A partir de las ecuaciones que modelan al vehículo, se llevó a cabo la identificación de las constantes del cuerpo libre, como ser el tensor de inercia, y también, la identificación de los actuadores, como ser el factor de torque y el factor de empuje.

En ocasiones, la dinámica de los actuadores es considerada despreciable frente a la dinámica del drone completo. Para esto, se presentó un ensayo de velocidad de actuadores y su respuesta al escalón.

A partir de los resultados del ensayo de velocidad de respuesta del sistema (figura 12), puede observarse que existen saltos pronunciados al cambiar el ciclo de actividad del PWM. Esto es debido en gran parte a la velocidad de respuesta del actuador, haciendo que la aproximación presentada en las ecuación (4), (5) y (6) no sean válida para cualquier sistema actuador-motor.

Finalmente, se propusieron dos bancos de pruebas para ensayar al sistema de control en forma independiente para cada grado de libertad antes del vuelo real. 


\section{REFERENCIAS}

Enrique D. Fliess,(1970) “Estabilidad”, tercera edición, Kapelusz S.A., Argentina, 165-204.

Ferdinand P. Beer y E. Russell Johnston Jr., (1997)“Mecánica vectorial para ingenieros: Estática”, sexta edición, McGraw-Hill, USA, 515-517.

Ferdinand P. Beer y E. Russell Johnston Jr., (1998)“Mecánica vectorial para ingenieros: Dinámica”, sexta edición, McGraw-Hill, USA, 1107-1114.

Duane Hanselman, (2006) "Brushless Permanent Magnet Motor Design", segunda edición, Magna Physics Publishing, USA, pp. 11-13.

S. Bouabdallah and R. Siegwart, (2007)“Full control of a quadrotor," en IROS '07 Proceedings of the International conference on Intelligent Robots and Systems, pp. 153-158.

Samir Bouabdallah, (2007)"Design and Control of Quadrotors with Application to Autonomous Flying”, Thesis (Ph. D. in Electrical Engineering), EPFL, Laussane Switzerland, 129.

Paul Edward Ian Pounds, (2007"Design, Construction and Control of a Large Quadrotor Micro Air Vehicle", Thesis (Ph. D. in Electrical Engineering), Australian National University, Australia, 171).

Guilherme Vianna Raffo, (2011)"Robust Control Strategies for a Quadrotor Helicopter", Thesis (Ph. D. in Electrical Engineering), Universidad de Sevilla, Sevilla - España, 267.

Daniel Martín Schermuk, (2012)“Diseño e Implementación de un Controlador para la Orientación de un QuadRotor”, Tesis (Tesis de grado para Ingeniería Electrónica), Universidad de Buenos Aires, Ciudad de Buenos Aires - Argentina, 111.

Alan Kharsansky, (2013)“Diseño e implementación de un sistema embebido de control de actitud para aeronaves no tripuladas”, Tesis (Tesis de grado para Ingeniería Electrónica), Universidad de Buenos Aires, Ciudad de Buenos Aires Argentina, 113.

Jesús Norberto Guerrero Tavares, (2014)“Implementación de un Algoritmo de Control para el Levantamiento de un Helicóptero de Cuatro Rotores", Tesis (Grado de Maestría en Ciencias en instrumentación y control automático), Universidad Autónoma de Querétaro, Querétaro - México, 98.

Rhudolabz Embedding Intelligence, (2018). 